\title{
Verifying Systems with Replicated Components in $\operatorname{Mur} \varphi^{*}$
}

\author{
C. Norris Ip and David L. Dill \\ Computer Systems Laboratory, Stanford University \\ Email: \{ip,dill\}@cs.stanford.edu
}

\begin{abstract}
We present an extension to the Mure verifier to verify systems with replicated identical components. Verification is by explicit state enumeration in an abstract state space where states do not record the exact numbers of components. Through a new datatype, called RepetitivelD, the user can suggest the use of such an abstraction to verify a system of fixed size. Mur $\varphi$ automatically checks the soundness of the abstract state graph, and automatically constructs the abstract state graph using the system description.

Using a simple run time check, Mur $\varphi$ can also determine if it can generalize the verification result of a system with fixed size to systems of larger sizes, including the system with infinite number of components.
\end{abstract}

\section{Introduction}

Finite-state systems such as cache coherence protocols, communication protocols or hardware controllers are often designed to be scalable, so that a description gives a family of different systems, each member of which has a different number of replicated identical components. It is therefore desirable to be able to verify the entire family of systems, independent of the exact number of replicated components.

The general problem of verifying systems with replicated components is known to be undecidable [AK86, GS92]. A number of approaches has been proposed for verifying particular classes of problems. Some of them use induction over the replicated components and require an invariant process or a network invariant [KMOS94, CG87, CGJ95, WL89]. Coming up with a proper invariant is not easy, and automatic generation of network invariants for certain classes of systems are restricted and expensive [RS93, BSV94, SG87, GS92].

There are also approaches that do not use induction. Shibata et al. [SHTO93] presented an algorithm to verify a simple telecommunication system with limited interaction between the processes. However, the class of problems they can verify is severely restricted. On the other hand, Graf [Gra94] has a more general method based on abstractions, which has been applied to a distributed cache memory, but it requires substantial manual effort to complete the proof.

The work described here is closely related to the methods by Lubachevsky [Lub84], Dijkstra [Dij85], and Pong et al. [PD95, PNAD95]. Lubachevsky verified a concurrent program by collapsing all reachable states into a fixed number of "metastates", in which the number of processes is represented by $N$ with an unspecified value. Dijkstra

\footnotetext{
* This research was supported by Semiconductor Research Corporation under contract 95-DJ-389 and by the Advanced Research Projects Agency through NASA grant NAG-2-891.
} 
used regular expressions to represent classes of similar states. Pong et al. used a set of repetition constructors to abstract away the exact number of components, for the verification of cache coherence protocols.

In this exposition, we consider systems with a collection of components, including fixed components and components that can be replicated from 1 to $n$ times. Many of these systems, such as cache coherence protocols and mutual exclusion algorithms, can be proved correct without modelling the precise number of replicated components. For example, suppose a multiprocessor has identical caches numbered 1 to 8 , and that a particular memory value is invalid in caches $1,2,3,5,6,7$ and and writable in cache 4 . The abstract state may record that more than zero caches are invalid, exactly one is writable, "forgetting" not only the number of processors in each state but also the ordering of the processors. Formally, the abstract state includes a mapping from component states to repetition constructors $\{0,1,+\}$, representing zero, exactly one, or more than zero (respectively) components in that component state. This abstraction is an approximation of the original state graph, which can be used for verification of invariants and $\forall \mathrm{CTL}$ model checking. The approximation is conservative: it never fails to report an error, but may report an error when none exists.

This approach has been used for the verification of several applications, but most of the existing work requires a lot of expertise from the user. For example, Pong and Dubois' method requires the user to write an executable description of the abstract behavior. This description is different from the concrete description used for specification or synthesis, so their method requires more work, and raises the question of whether the concrete and abstract descriptions are consistent.

To reduce the amount of user effort, we incorporate this abstraction into our verification system, Mur $\varphi$. We provide an extension to the existing high-level language in which the user can easily specify a protocol in its concrete form. The extension is a new datatype, called RepetitiveID, which can be used to represent the indices of the replicated components. The Mur $\varphi$ compiler can automatically detect whether the datatype is used in a way that admits the use of repetition constructors in verification. If so, it automatically verify the system using the abstract state space, instead of the concrete state space.

Furthermore, we also extend previous work so that the abstraction can be used to verify systems of fixed sizes, even when verification for unbounded sizes is infeasible, resulting substantial reductions in the state explosion. Through a simple run-time check, Mur $\varphi$ can determine automatically if it can generalize the verification result to systems of larger sizes.

A key problem in verifying in an abstract state space is how to generate the successors of an abstract state. We solve this problem by selecting up to two concrete states represented by an abstract state, and constructing the abstract successors from the concrete successors of these concrete states. Heurstics for an efficient construction of the abstract state space are also presented.

\section{Modifications to Mur $\varphi$}

\subsection{The Mur $\varphi$ Verification System}

The basic Mur $\varphi$ Verification System [DDHY92] consists of the Mur $\varphi$ compiler and the Mur $\varphi$ description language. The Mur $\varphi$ description language is a high-level programming language for the description of finite-state asynchronous concurrent systems. The Mur $\varphi$ 
compiler generates a $\mathrm{C}++$ program for a $\mathrm{Mur} \varphi$ program, which exhaustively generates the reachable states, checking for error conditions and deadlocks.

A Mur $\varphi$ program consists of four parts: declarations, transition rules, start state generation rules, and invariant descriptions. Examples of Mur $\varphi$ programs are shown in Figs. 1 and 2.

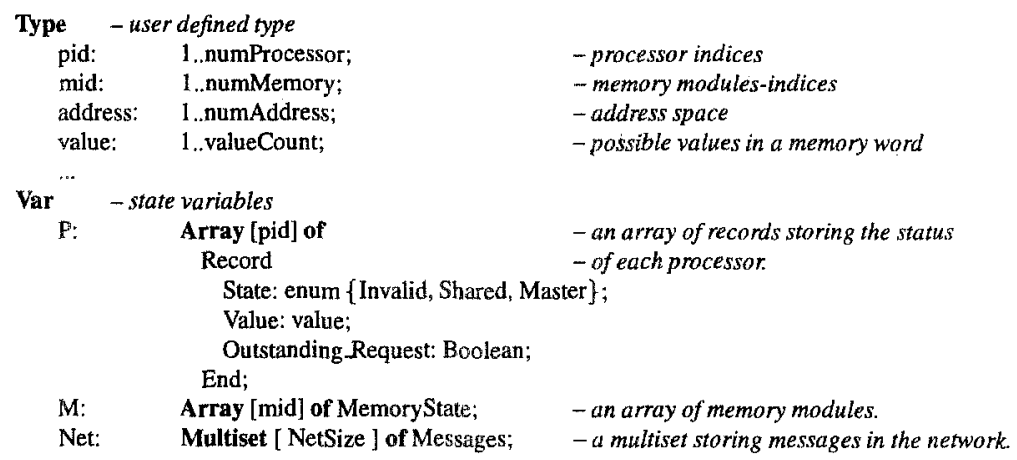

Fig. 1. An Example of state variable declarations in Mur $\varphi$

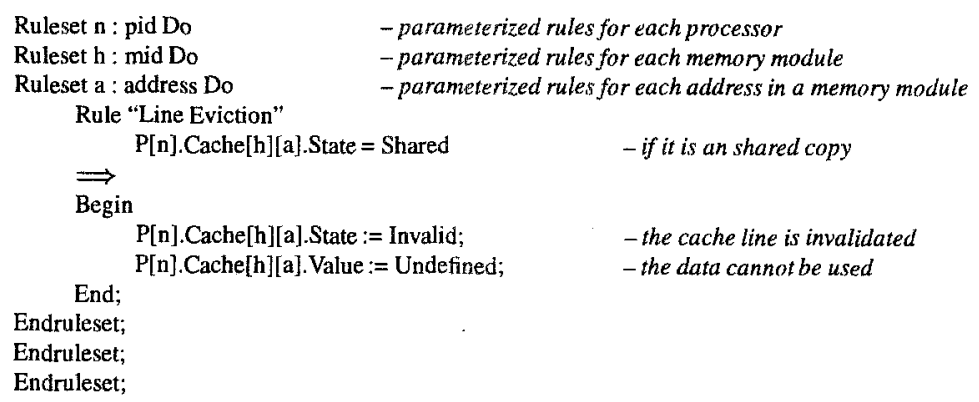

Fig. 2. An Example of transition rules in Mury

A system state is specified by the values of the global variables. The rules are conditional actions (guarded commands). As a Mure description executes, a rule is chosen nondeterministically and executed, generating a new system state (since it assigns new values to the variables). Although a rule may consist of arbitrarily complex operations, it is executed atomically, without interference from other rules in the description. Hence, the use of Mur $\varphi$ leads to an asynchronous, interleaving model of concurrency in which different parts of the system interact via shared variables.

The types of variables are mostly conventional finite datatypes found in high level languages: arrays, records, integer subranges, Booleans and enumerations. Unlike conventional languages, there is also a special "undefined" value for each datatype. There are some non-traditional types as well, such as scalarsets [ID93a] and multisets. The scalarset type is a finite set of values, similar to a subrange except that their use is 
stant for the number of components (say Compcount), and defining a subrange CompID: 1 . CompCount for the indices of components. The local states. of the components are stored in an array indexed by CompID. The rules describing the components are enclosed in a ruleset with a CompID parameter which represents the component to which the rule belongs. Using this convention, Mur $\varphi$ descriptions become scalable, meaning that the number of replicated components can be changed simply by modifying Compcount.

\subsection{The RepetitiveID Type}

We add a new datatype to Mur $\varphi$, called RepetitiveID. The RepetitivelD is a restricted subclass of a conventional subrange (in fact, it is a scalarset), which should be used for the indices of replicated identical components, such as processors in a multiprocessor. For example, we can change the subrange 1 . numProcessor to RepetitiveID (numProcessor) to hint to the verifier to verify the system in the smaller abstract state space. Mure automatically checks that certain restrictions are satisfied so that the verification is sound. Since a member $i$ of the RepetitiveID type is used as the name of a component in the description, it is natural to identify $i$ and the component, and refer to "component $i$ " below.

A value of RepetitiveID type can be assigned to variables, tested for equality with other values, used as an array index, or bound in a RuleSet or for loop. There are six restrictions on the use of RepetitiveID. In spite of the restrictions, RepetitiveID can be used to model a wide range of systems, including bus-based multiprocessor cache coherence protocols [PD95], network-based cache coherence protocols with a central or distributed directory [PNAD95, DDHY92, LLG ${ }^{+}$90].

Intuitively, our goal is to isolate the parts of the state corresponding to the replicated components into a single array indexed by the RepetitiveID type. If two components $i$ and $j$ have identical component states, we would like the transition rule to produce a successor state where $i$ and $j$ have identical component states, also. There is one exception: we allow the rule to have one "special" component, whose component state is treated differently from other component states, even if they are otherwise identical. An example of where this is useful is mutual exclusion: many components may be in identical states, waiting for a resource, but only one (the special one) will obtain it.

The first two restrictions make it possible to separate the component states from other parts of the state.

\section{The Mure program has at most one RepetitivelD type.}

2. The elements of a symmetric array cannot contain another array with RepetitiveID index type. A symmetric array is an array indexed by a scalarset or RepetitiveID type, or a multiset.

We illustrate the subsequent definitions with an example. Consider a central-directorybased cache coherence protocol whose state includes: an array of local processor control states; a multiset of messages representing a communications network; a memory where each memory line has an owner field pointing to a processor that has a writable copy of the line, along with the data in the memory line. The messages in the network have to and from fields, which can be processor indices or a value representing the memory itself. 
With these restrictions, the following definition characterizes the parts of the state that "belong" to a replicated component.

Definition 1. The component state of the component $i$ includes all the state variables satisfying:

- for every array $A$ indexed by the components, the element $A[i]$. (In the example, the local control state of processor $i$ becomes part of the component state for $i$.)

- for every multiset $M$ that is not assigned to a component state by the previous case, the elements of $M$ containing $i$ and no other components. (In the example, the messages between the memory and processor $i$ become part of the component state for $i$.)

In our example, the memory value (which does not contain a component index), the owner field (which is not in a multiset), and messages from one processor to another (which contain two component indices) are not included in any component states.

Definition 2. A component, $i$, is abstractable if it contains all instances of $i$ occuring in the global state, and contains no other component indices.

In our example, a processor $i$ would not be abstractable if the owner field had the value $i$, or if there were a message between $i$ and another processor $j$.

In the rest of the paper, we regard the state as being a pair $\left(s,\left[r_{1}, \ldots, r_{k}\right]\right):\left[r_{1}, \ldots, r_{k}\right]$ is an array of component states indexed by the abstractable components, and $s$ an assignment to the rest of the state variables. The component states for components $i$ and $j$ are considered to be the same if the only difference between corresponding variables is that variables in component $i$ have the value $i$ and variables in component $j$ have the value $j$.

There are four restrictions on the use of RepetitivelDs in Mur $\varphi$ to ensure the soundness of this verification method. Although we have made these as simple as we can, some are still quite technical.

3. No "symmetry-breaking" operations [ID93a]. There are no literal constants in the type; arithmetic operations are not allowed; comparisons such as $<$ are not allowed.

4. Bindings of the RepetitivelD type in RuleSet constructs may not be nested.

5. Bindings of the RepetitiveID type in for statements and forall expressions may not be nested. The variables written by each iteration of a for statement on the RepetitiveID must be disjoint. In particular, a forall expression on the RepetitiveID is not allowed in the body of a for statement on the RepetitiveID.

6. If a variable in the state has the RepetitivelD type, and its value is $i$ for some abstractable components, the variable may not be used to index an array with RepetitivelD index type.

Intuitively, the symmetry restriction makes sure that the components can be reordered arbitrarily without changing the behavior of the systems. The remaining restrictions ensure that transition rules treat identical component states identically, except for at most one special component. 


\section{Verification Using Repetition Constructors}

\subsection{Abstract States Using Repetition Constructors}

Once we have isolated the abstractable components as in the previous section, it is possible to abstract away from the exact numbers of each component state by using the repetition constructors 0,1 , and + .

Definition 3 Abstract State. An abstract state is similar to a concrete state except that the array of abstractable components is replaced with a mapping of each possible component state to one of the repetition constructors 0,1, or + .

Abstract states are written in the form $\left(s,\left\{q_{1}^{e_{1}}, \ldots, q_{k}^{e_{k}}\right\}\right)$, where each $e_{i}$ is 1 or + (when the constructor is 0 , the component state is omitted).

A concrete state $a=\left(s,\left[r_{1}, \ldots, r_{z}\right]\right)$ is represented by an abstract state $A=$ $\left(s,\left\{q_{1}^{e_{1}}, \ldots, q_{k}^{e_{k}}\right\}\right)$ if the following conditions are satisfied:

- $e_{i}=+$ if $q_{i}$ occurs in $\left[r_{1}, \ldots, r_{z}\right]$ two or more times;

- $e_{i}=1$ or $e_{i}=+$ if $q_{i}$ occurs in $\left[r_{1}, \ldots, r_{z}\right]$ exactly once;

- a component state does not appear in $\left[q_{1}, \ldots, q_{k}\right]$ if it does not appear in $\left[r_{1}, \ldots, r_{z}\right]$.

The abstract states are partially ordered: $\left(s,\left\{q_{1}^{e_{1}}, \ldots, q_{k}^{e_{k}}\right\}\right) \leq\left(s,\left\{q_{1}^{e_{1}^{\prime}}, \ldots, q_{k}^{e_{k}^{\prime}}\right\}\right)$ if and only if $e_{i}=+$ implies $e_{i}^{\prime}=+$. In this case, $\left(s,\left\{q_{1}^{e_{1}^{\prime}}, \ldots, q_{k}^{e_{k}^{\prime}}\right\}\right)$ is said to cover $\left(s,\left\{q_{1}^{e_{1}}, \ldots, q_{k}^{e_{k}}\right\}\right)$. The notation $a \in A$ is used to indicate that $A$ represents $a$. The set of abstract states representing a particular concrete state has a unique minimum element in this order; the abstracting function abs used in our verifier maps a concrete state to its minimum abstract representative.

In many cases, it is useful to maintain in the abstract state the total number of replicated components, while forgetting exactly how many components are in each component state.

Definition 4 Restricted Abstract State. A restricted abstract state is an abstract state paired with a number representing the total number of replicated components. nents.

We write $\left.\left(s, q_{1}^{e_{1}}, \ldots, q_{k}^{e_{k}}\right)\right|_{n}$ to represent the restricted abstract state with $n$ compo-

\subsection{The Basic On-The-Fly Algorithm}

We can construct the abstract state graph for a Mur $\varphi$ program with a RepetitiveID type using an on-the-fly algorithm,

First of all, $\mathrm{C}++$ code for the abstraction function abs is generated by the Mur $\varphi$ compiler. The start states of the abstract state graph are generated by using this function to abstract the concrete start states.

Given an abstract state, the verifier needs to generate all its successors in the abstract state graph. Because of the restrictions of the RepetitiveID, the verifier can always find a small number of concrete states that can be used to find the successors to the abstract state. The choice of concrete states depends on the abstract state, and on the nature of the concrete transition functions. 
Given $\left(s,\left\{q_{1}^{e_{1}}, \ldots, q_{k}^{e_{k}}\right\}\right)$, and a transition $t$, there are three possible situations:

1. There is no special component for the transition $t$, or the special component is not abstractable, i.e., it belongs to $s$.

2. The special component has repetition constructor 1 .

3. The special component has repetition constructor + .

For brevity, we discuss only the third, most difficult, case in detail. Suppose $i$ is the special component for $t$, and $i$ has component state $q_{i}$. The restrictions of the RepetitiveID allow a transition to have different effects on the special component and the other components with the same component state. Therefore, we split our abstract state into two concrete states $\left(s,\left[q_{1}, \ldots, q_{i-1}, q_{i}, q_{i+1}, \ldots, q_{k}\right]\right)$ and $\left(s,\left[q_{1}, \ldots, q_{i}, q_{i}, \ldots, q_{k}\right]\right)$, as shown in Fig. 4.

If the successor of $\left(s,\left[q_{1}, \ldots, q_{i-1}, q_{i}, q_{i+1}, \ldots, q_{k}\right]\right)$ is $\left(s^{\prime},\left[r_{1}, \ldots, r_{i-1}, r, r_{i+1}, \ldots, r_{k}\right]\right)$, we can convert it back to an abstract state by restoring the repetition constructors $\left(e_{1}, \ldots, e_{i-1}, 1, e_{i+1}, \ldots, e_{k}\right)$ to get $\left(s^{\prime},\left\{r_{1}^{e_{1}}, \ldots, r_{i-1}^{e_{i}-1}, r^{1}, r_{i+1}^{e_{i}+1}, \ldots, r_{k}^{e_{k}}\right\}\right)$. To make sure it is an legal abstract state, we may have to re-partition if the component with state $r$ is no longer abstractable, and to combine any identical component states $r_{i}$ and $r_{j}$. Similarly another abstract successor is generated from $\left(s,\left[q_{1}, \ldots, q_{i}, q_{i}, \ldots, q_{k}\right]\right)$ with repetition constructors $\left(e_{1}, \ldots, 1,+, \ldots, e_{k}\right)$.

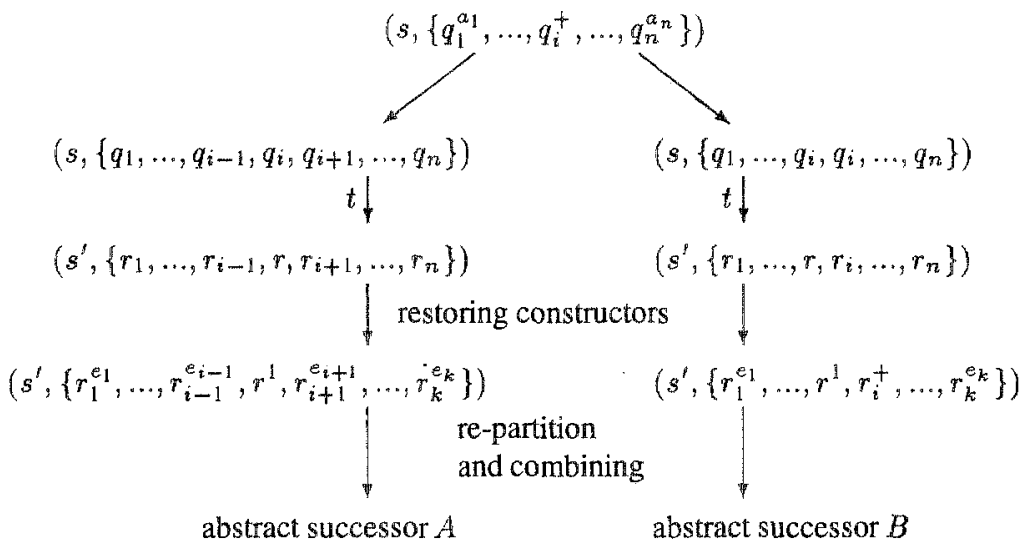

Fig. 4. Transition $t$ With Special Component in State $q_{i}$ and Constructor + .

Because of the restrictions of the RepetitiveID, the abstract state graph generated this way has the following property:

Property 1 Given two abstract states $A$ and $B$, if there exists concrete state $a \in A$ and $b \in B$ such that $(a, b)$ is a transition in the original state graph, $(A, B)$ is a transition in the abstract state graph.

The abstract start states represent all concrete start states, and the abstracting function abs maps error to error. Therefore, it can easily be proved by induction that for every state reachable from a start state in the concrete state graph, there is an abstract 
representative reachable from an abstract start state. This can be easily proved by induction.

It follows from this result and $\left[\mathrm{BBG}^{+93}\right]$ that if the abstract state graph satisfies a $\forall$ CTL formula $f$, the concrete state graph also satisfies $f$. Because the abstract state graph is an approximation, it may result in reports of non-existent errors and cannot be used for verification of deadlock-freedom (there is no reachable state with only itself as successor) or $\exists$ CTL model checking.

The algorithm implemented in Mur $\varphi$ uses the restricted abstract states. The Mur $\varphi$ program is required to specify the number of replicated components, $n$, and the Mur $\varphi$ verifier restricts all abstract states to size $n$. There are two situations when a restricted abstract state represents no concrete states, and therefore, is discarded. One is when it only represents concrete states with number of replicated components fewer than $n$, such as in the case of the restricted abstract state $\left.\left(s,\left\{q_{1}^{1}, q_{2}^{1}\right\}\right)\right|_{3}$. The other situation is when it only represents concrete states with number of replicated components more than $n$, such as in the case of the restricted abstract state $\left.\left(s,\left\{q_{1}^{+}, q_{2}^{+}\right\}\right)\right|_{1}$.

Furthermore, in some cases, the restricted abstract state represents the same set of concrete states as a similar restricted abstract state with the 1 constructor only. Mur $\varphi$ automatically converts such states to a restricted abstract state with the 1 constructor only. For example, the restricted abstract states $\left.\left(s,\left[q_{1}^{1}, q_{2}^{+}\right]\right)\right|_{2}$ and $\left.\left(s,\left[q_{1}^{+}, q_{2}^{1}\right]\right)\right|_{2}$ is the same as $\left.\left(s,\left[q_{1}^{1}, q_{2}^{1}\right]\right)\right|_{2}$, and Mur $\varphi$ automatically converts both of them to $\left.\left(s,\left[q_{1}^{1}, q_{2}^{1}\right]\right)\right|_{2}$.

During the verification process $s_{\mathrm{s}}$ if the discarded abstract state only represents concrete states with fewer components than $n$, and no restricted abstract state is converted to one with the 1 constructor only, the abstract state graph obtained is the same as that for systems of larger sizes. We call this state graph the saturated state graph. The saturated state graph represents the behavior of all systems with sizes $n$ or larger. The verification result is therefore valid for all systems of size $n$ or larger.

With the restricted abstract state graph, the verifier won't attempt to solve the problem for arbitrary sizes if it is unsolvable or if the abstract state graph for arbitrary system sizes is too large for us to verify.

\subsection{The Efficient On-The-Fly Algorithm}

The algorithm presented in the previous subsection is very inefficient, because it is wasteful to have two comparable states in the table of previously examined states. For example, $\left(s,\left\{q_{1}^{1}, q_{2}^{+}\right\}\right)$is redundant in the set of previously examined states when $\left(s,\left\{q_{1}^{+}, q_{2}^{+}\right\}\right)$is also in the set. A more efficient algorithm can be implemented as shown in Fig. 5. Two heuristics are presented in this section to reduce the time for checking whether a state is maximal in the set of previously generated states and to reduce the number of non-maximal states generated.

Checking if a State is Maximal For every abstract state generated, we check whether it is covered by a previously examined state, and remove any previously examined state that is covered by it. Pong et al. use a linear search on all previously examined states to do this.

If an abstract state $p$ covers another abstract state $p^{\prime}, p$ and $p^{\prime}$ can only differ in the 1 and + constructors on their component states. To store the abstract states, the hash function does not distinguish between 1 and + constructors. The states hashing to the same location are searched linearly to see if any cover or are covered by the current state. 


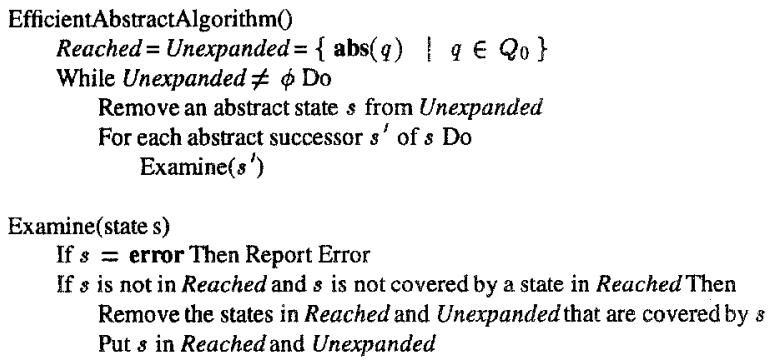

Fig. 5. The Efficient On-the-fly Algorithm Using the Abstract State Space

In practise, the lists are very short. In the industrial cache coherence protocol presented in Section 4, the maximum length is 6 . When an abstract state is hashed into a location with a list of constructor arrays, the average number of states compared is fewer than 1.05. This method does not work well for the original scheme of Pong et al., because they have a fourth constructor, *, meaning "zero or more states".

Reducing the Number of Non-Maximal States Although an abstract model has very few states, many non-maximal states are temporarily stored and expanded. We have found that for simple depth first search (DFS) and breadth first search (BFS), more than $75 \%$ of the time is spent searching non-maximal states.

Instead of using simple DFS or BFS, we use a best-first strategy, where "best" is defined as the greatest number of + constructors. The abstract state that represents more concrete states are expanded to find its successors first, because its successors are more likely to be maximal states. For the industrial cache coherence protocol presented in Section 4, we are able to reduce the number of non-maximal states examined from 106,528 ( 3 times more than the number of maximal states) down to 3,527 (fewer than $10 \%$ of the maximal states) in a 9-processor system. No extra memory is required to store the non-maximal states, and the verification is more than three times faster.

Pong also has mentioned similar strategy in his thesis [Pon95], however, because of his choice of repetition constructors, it does not help much in reducing the number of non-maximal states.

\section{Practical Results}

The abstraction with the repetition constructors can be combined easily with the other two reduction strategies implemented in Mur $\varphi$ : symmetry reduction [ID93a, ID93b] and reduction by reversible rules [ID96].

We present in this section the verification results for an industrial cache coherence protocol (ICCP), using the Mur $\varphi$ verification system. This protocol is a typical centraldirectory-based cache coherence protocol, as described in [DDHY92]. Because of data forwarding, some replicated components in some states are not abstracted by the repetition constructors. However, since the extent of forwarding is limited, we are still able to verify it for arbitrary system sizes, as shown in Table 1.

As we increase the size of the system, the size of the abstract state graph increases accordingly until it becomes saturated. After the verification of a system of 14 processors, 
Table 1. Results for the Verification of an Industrial Cache Coherence Protocol

\begin{tabular}{|c|c|c|c|c|c|c|c|}
\hline \# of processors (ICCP) & 3 & 4 & 5 & 6 & 7 & 8 & $9]$ \\
\hline size (unordered network) & 10,077 & 247,565 & - & - & - & - & - \\
\hline size (sym. only) & 1,781 & 11,814 & 68,879 & 358,078 & - & - & - \\
\hline size (sym./rep.) & 1,770 & 11.206 & 57,790 & 257,692 & - & - & - \\
\hline size (sym./rev.) & 434 & 1,760 & 6,021 & 18,118 & 49,045 & 121,302 & \\
\hline size (sym./rev/rep.) & 427 & 1,590 & 4,542 & 10,587 & 19,485 & 28,927 & 35,515 \\
\hline time (unordered network) & $5.1 \mathrm{~s}$ & $205 \mathrm{~s}$ & - & - & - & - & - \\
\hline time (sym. only) & $24 \mathrm{~s}$ & $28 \mathrm{~s}$ & $349 \mathrm{~s}$ & $3,762 \mathrm{~s}$ & - & - & \\
\hline time (sym./rep.) & $4.4 \mathrm{~s}$ & $49 \mathrm{~s}$ & $497 \mathrm{~s}$ & $4,555 \mathrm{~s}$ & - & - & \\
\hline time (sym./rev.) & $2.1 \mathrm{~s}$ & $13 s$ & $98 \mathrm{~s}$ & $615 \mathrm{~s}$ & $3,283 \mathrm{~s}$ & $12,801 \mathrm{~s}$ & \\
\hline time (sym./rev./rep.) & $3.3 \mathrm{~s}$ & $27 \mathrm{~s}$ & $167 \mathrm{~s}$ & $811 \mathrm{~s}$ & $3,265 \mathrm{~s}$ & $7,593 \mathrm{~s}$ & $17,477 \mathrm{~s}$ \\
\hline \# of processors (ICCP) & 10 & 11 & 12 & 13 & & & 4 and up \\
\hline size (sym./rev./rep.) & 38,146 & 38,485 & 38,329 & 38,269 & & & 38,269 \\
\hline time (sym./rev./rep.) & $29,871 \mathrm{~s}$ & $37,903 \mathrm{~s}$ & $43,352 \mathrm{~s}$ & $48,410 \mathrm{~s}$ & & & $49,932 \mathrm{~s}$ \\
\hline
\end{tabular}

Mur $\varphi$ was able to detect automatically that the abstract state graph is the same for systems with 15 processor or more. The saturated model has 38,269 states and is valid for 14 processors or more. This phenomenon is very similar to the data saturation phenomenon reported in [ID93a, ID93b].

\section{Acknowledgement}

We would like to thank Fong Pong for the discussion on the symbolic state model, Ganesh Gopalakrishnan, Seungjoon Park, Ulrich Stern, and Han Yang for their valuable feedback during the writing of this paper.

\section{References}

[AK86] Krzysztof R. Apt and Dexter C. Kozen. Limits for automatic verification of finitestate concurrent systems. Information Processing Letters, 22:307-309, 1986.

$\left[\mathrm{BBG}^{+}\right.$93] A. Bouajjani, S. Bensalem, S. Graf, C. Loiseaux, and J. Sifakis. Property preserving abstractions for the verification of concurrent systems. Formal Methods in System. Design, 1993.

[BSV94] F. Balarin and A.L. Sangiovanni-Vincentelli. On the automatic computation of network invariants. 6th Int'l Conf. on Computer-Aided Verification, June 1994.

[CC92] Patrick Cousot and Radhia Cousot. Abstract interpretation and application to logic programs. Technical report, Ecole Polytechnique, Laboratoire d'Informatique, 1992.

[CG87] E.M. Clarke and O. Grumberg. Avoiding the state explosion problem in temporal logic model checking algorithms. Proceedings of the 6th Annual ACM Symp. on Priniciple of Distributed Computing, 1987.

[CGJ95] E.M. Clarke, O. Grumberg, and S. Jha. Verifying parameterized networks using abstraction and regular languages. CONCUR'95, 1995.

[DDHY92] David L. Dill, Andreas J. Drexler, Alan J. Hu, and C. Han Yang. Protocol verification as a hardware design aid. Int'l Conf. on Computer Design: VLSI in Computers and Processors, 1992. 
[Dij85] E.J. Dijkstra. Invariance and nondeterminacy. In Mathematical Logic and Programming Languages. Prentice-Hall, 1985.

[GL93] S. Graf and C. Loiseaux. A tool for symbolic program verification and abstraction. 5th Int'l Conf. on Computer-Aided Verification, April 1993.

[Gra94] Susanne Graf. Verification of a distributed cache memory by using abstractions. 6th Int'l Conf. on Computer-Aided Verification, 1994.

[GS92] S.M. German and A.P. Sistla. Reasoning about systems with many processes. Journal of Association for Computing Machinery, 39(3):675-735, 1992.

[ID93a] C. Norris Ip and David L. Dill. Better verification through symmetry. 11th Int'l Symp. on Computer Hardware Description Languages and Their Applications, 1993. Extended version with complete proofs and semantic analysis to appear in Formal Methods in System Design.

[ID93b] C. Norris Ip and David L. Dill. Efficient verification of symmetric concurrent systems. Int'l Conf. on Computer Design: VLSI in Computers and Processors, 1993.

[ID96] C. Norris Ip and David L. Dill. State reduction using reversible rules. 33rd Design Automation Conference, June 1996.

[KMOS94] Robert P. Kurshan, Michael Merritt, Ariel Orda, and Sonia R. Sachs. A structural linearization principle for processes. Formal Methods in System Design, 5, 1994.

[LLG ${ }^{+}$90] Daniel Lenoski, James Laudon, Kourosh Gharachorloo, Anoop Gupta, and John Hennessy. The directory-based cache coherence protocol for the dash multiprocessor. 17th Int'l Symp. on Computer Architecture, 1990.

[Lub84] Boris D. Lubachevsky. An approach to automating the verification of compact parallel coordination programs I. Acta Informatica, 21:125-169, 1984.

[PD93] F. Pong and M. Dubois. Correctness of a directory-based cache coherence protocol: Early experience. 5th Symp. on Parallel Distributed Processing, 1993.

[PD95] F. Pong and M. Dubois. A new approach for the verification of cache coherence protocols. IEEE Transactions on Parallel and Distributed Systems, 6(2), 1995.

[PNAD95] F. Pong, A. Nowatzyk, G. Aybay, and M. Dubois. Verifying distributed directorybased cache coherence protocols: S3.mp, a case study. EurPar'95.

[Pon95] Fong Pong. Symbolic State Model: A New Approach for the Verification of Cache Coherence Protocols. PhD thesis, University of Southern California, 1995.

[RS93] June-Kyung Rho and Fabio Somenzi. Automatic generation of network invariants for the verification of iterative sequential systems. Sth Int'l Conf. on Computer-Aided Verification, June 1993.

[SG87] A.P. Sistla and S.M. German. Reasoning with many processes. Symp. on Logic in Computer Science, 1987.

[SHTO93] Kenji Shibata, Yutaka Hirakawa, Akira Takura, and Tadashi Ohta. Reachability analysis for specified processes in a behavior description. IEICE Transaction on Communication, E76-B(11), November 1993.

[WL89] Pierre Wolper and Vinciane Lovinfosse. Verifying properties of large sets of processes with network invariants. In Automatic Verification Methods for Finite State Systems, volume 407 of LNCS, Springer-Verlag, 1989. 